\title{
Techniques
}

\section{Anaplastic Lymphoma Kinase Immunocytochemistry on Cell-Transferred Cytologic Smears of Lung Adenocarcinoma}

\author{
Chen Zhang ${ }^{a}$ Melissa L. Randolph ${ }^{b}$ Kelly J. Jones ${ }^{b}$ Harvey M. Cramer \\ Liang Cheng ${ }^{\mathrm{a}}$ Howard H. Wu ${ }^{\mathrm{a}}$ \\ ${ }^{a}$ Department of Pathology and Laboratory Medicine, Indiana University School of Medicine, and ${ }^{\mathrm{b}}$ Department of \\ Cytopathology, Indiana University Health, Indianapolis, Ind., USA
}

\section{Key Words}

Anaplastic lymphoma kinase · Immunocytochemistry · Cell transfer

\begin{abstract}
Background: Anaplastic lymphoma kinase (ALK) immunohistochemical staining on formalin-fixed paraffin-embedded tissue or cell blocks (CB) has been reported as an effective alternative to fluorescence hybridization in situ (FISH) for the detection of $A L K$ gene rearrangement. However, $C B$ frequently lack adequate cellularity even when the direct smears are cellular. This study aims to assess the utility of ALK immunocytochemical (ICC) staining on direct smears using the cell transfer (CT) technique for the detection of $A L K$ rearrangement. Methods: Fine-needle aspiration (FNA) cases of lung adenocarcinoma in which the ALK status had been determined by FISH on CB or a concurrent biopsy were identified. ICC staining for ALK was performed on alcohol-fixed Papanicolaou-stained direct smears using the $C T$ technique. ALK immunoreactivity was evaluated using a modified semiquantitative scale. Results were compared with those of FISH. Results: A total of 47 FNA specimens were included. Five of 7 FISH-positive cases showed positive ALK ICC staining (71.4\%), and 39 of $40 \mathrm{FISH}$-negative cases were negative
\end{abstract}

on ALK ICC staining (97.5\%). The overall correlation between ALK ICC and FISH was $93.6 \%$. Conclusion: ICC performed on FNA smears using the $C T$ technique is an alternative method for the assessment of $A L K$ rearrangement, especially when $\mathrm{CB}$ lack adequate cellularity.

(c) 2015 S. Karger AG, Basel

\section{Introduction}

Anaplastic lymphoma kinase (ALK) gene rearrangements represent the primary oncogenic driver in 3-5\% of patients with non-small cell lung cancers (NSCLC), especially adenocarcinomas, with the echinoderm microtubule-associated protein-like 4 (EML4) gene being the most frequent translocation partner $[1,2]$. The ALK inhibitor crizotinib has been associated with a response rate of over $60 \%$ in ALK-rearranged advanced NSCLC [3, 4], as shown by multicenter clinical trials. ALK status is now a required study for NSCLC, recommended by multiple societies including the College of American Pathologists (CAP) [5]. In previous clinical trials, the ALK status has been determined by fluorescence in situ hybridization (FISH) using the Vysis break-apart probe set (Abbott Molecular, Des Plaines, Ill., USA), which to date is the

\section{KARGER 125}

C 2015 S. Karger AG, Base

$0001-5547 / 15 / 0592-0213 \$ 39.50 / 0$

E-Mail karger@karger.com

www.karger.com/acy
Correspondence to: Dr. Howard H. Wu

Department of Pathology and Laboratory Medicine Indiana University School of Medicine

$350 \mathrm{~W}$ 11th Street, Indianapolis, IN 46202 (USA)

E-Mail hhwu@indiana.edu 
only diagnostic assay licensed for this purpose by the US Food and Drug Administration (FDA) [6].

The main advantage of the FISH method is the possibility of detecting all types of ALK rearrangements known to date. However, specialized techniques and training are required for the implementation and interpretation of ALK FISH testing. Significant interobserver variability has been shown even in the hands of experienced specialists [7]. Additionally, FISH as a screening assay is relatively expensive for most clinical settings. In contrast, immunohistochemistry (IHC) is relatively inexpensive, fast, and familiar to most pathologists. Zhou et al. [8] studied ALK IHC in 410 resected lung adenocarcinomas and compared the results to corresponding FISH assays. They found that all 333 IHC-negative cases were negative by FISH, and all $28 \mathrm{FISH}$-positive cases were positive by IHC. A similar study by Paik et al. [9] using 465 resected NSCLC also showed that the sensitivity and specificity of IHC compared to FISH was 100 and $95.8 \%$, respectively. CAP guidelines recommend using a sensitive ALK IHC as a screening test for ALK rearrangement on formalinfixed paraffin-embedded (FFPE) tissue [5].

With the advance of imaging-assisted biopsy techniques and fine-needle aspiration (FNA) cytology, the pathological diagnosis of lung cancer is more commonly done preoperatively on FNA material. The ALK FISH and IHC protocols used for FFPE tissue specimens can be used on cell blocks (CB) for immunocytochemistry (ICC) assays. However, although we perform rapid on-site evaluations on all FNA done at our institution and typically collect multiple dedicated passes for $\mathrm{CB}$ in lung cancer patients, the $\mathrm{CB}$ still sometimes lack adequate cellularity for ALK testing even when the direct smears are cellular. As an alternative approach, if conventional CB lack adequate cellularity, ICC can be performed on cell-transferred (CT) direct smears and multiple immunostains can be applied to a single direct smear using this technique $[10-13]$. The aim of this study is to assess the reliability of the CT technique for ICC assessment of the ALK status of aspirates of primary and metastatic lung adenocarcinomas.

\section{Materials and Methods}

This study was approved by the Institutional Review Board of Indiana University. A computerized search of the cytopathology and surgical pathology archives was performed. FNA cases diagnosed as primary or metastatic lung adenocarcinoma in which the ALK status had been previously evaluated by FISH on a CB or a concurrent surgical biopsy were identified over a period of 33 months (September 2011 through May 2014). The search yielded a total of 47 FNA specimens including FNA from 26 primary lung lesions and 21 metastatic lesions (lymph node, $\mathrm{n}=15$; bone, $\mathrm{n}=2$; liver, $\mathrm{n}=2$; chest wall, $\mathrm{n}=1$, and adrenal gland, $\mathrm{n}=1$ ). Of these, formalin-fixed core biopsies were used for FISH in 8 cases and formalin-fixed CB were used in 39 FNA cases.

The ethanol-fixed, Papanicolaou-stained direct smears from each case were reviewed, and the most cellular slide was chosen for $\mathrm{CT}$. The CT technique was performed as described previously [13]. Briefly, after removal of the coverslip using xylene, a thin layer of Mount-Quick medium (Daido Sangyo Co. Ltd., Japan) was spread uniformly over the wet slide to entirely cover the cellular material. The slide was then placed in a $60^{\circ} \mathrm{C}$ heated oven for $3 \mathrm{~h}$ or longer until the medium hardened. The cellular areas (at least 200 tumor cells) to be transferred were then marked using a permanent marker. After incubation in a $50^{\circ} \mathrm{C}$ water bath for $2 \mathrm{~h}$, the mounting medium with the embedded cells was slowly peeled off the slide using forceps and a scalpel. The marked area was cut off and placed on a charged glass slide. The slide was dried in a $60^{\circ} \mathrm{C}$ oven for at least $2 \mathrm{~h}$. The dried slide was washed in 4 exchanges of xylene ( 15 min each) to remove the mounting media, and it then was rehydrated with 2 exchanges of absolute alcohol, 2 exchanges of 95\% alcohol, and 2 exchanges of deionized water (15 min each). The slide was stored in deionized water until ICC was performed.

ICC for ALK was performed on the CT slides using the primary rabbit monoclonal anti-ALK antibody D5F3 (Cell Signaling Technology, Billerica, Mass., USA) with a Dako EnVision detection kit. No modifications were made to the immune-staining process on the CT slides compared to formalin-fixed tissue. The results were scored by 2 independent observers (C.Z. and H.H.W.) using modified semiquantitative graded criteria described previously [8] (table 1). ICC results were excluded from this study if the CT slide contained less than 200 cells. ICC results were compared with those of FISH performed on the correlating CB or core biopsy.

\section{Results}

The results of ICC on the CT smears and the results of FISH on correlating formalin-fixed CB or core biopsy are summarized in table 2. All 47 FNA specimens were adequately cellular for evaluation. The correlating FISH studies showed that 40 were negative and 7 were positive for $A L K$ rearrangement.

Five of 7 FISH-positive cases (71.4\%) showed positive ALK ICC staining, with 4 cases showing $3+$ staining (fig. 1) and 1 case showing 2+ staining. Two FISH-positive cases were negative on ICC (score 1+; fig. 2), and the concurrent core biopsy and $\mathrm{CB}$ of the 2 cases were also negative for D5F3 immunostaining (score 0). Both falsenegative cases showed borderline cellularity ( 200 tumor cells) and a relatively low percentage of tumor cells with ALK rearrangements on the FISH test (20 and 22\%). 

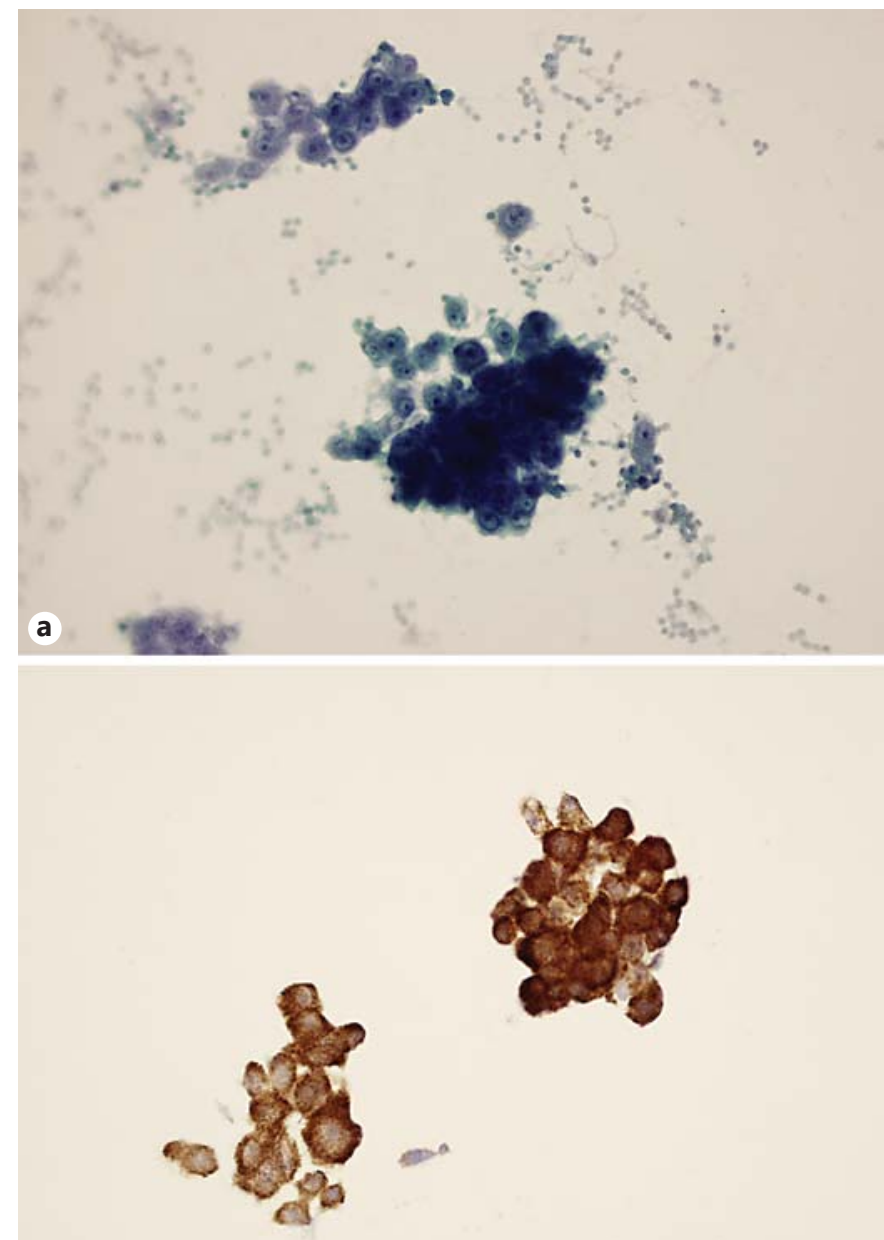

b

Fig. 1. Representative images of cases that were positive on ALK ICC and FISH. a Papanicolaou-stained direct smears before CT. b ALK ICC staining using the D5F3 antibody. Original magnification: $\times 400$.

Table 1. Scoring system for ALK by ICC

\begin{tabular}{lll}
\hline Score & Staining pattern & $\begin{array}{l}\text { ALK } \\
\text { status }\end{array}$ \\
\hline 0 & No staining & Negative \\
\hline $1+$ & $\begin{array}{l}\text { Faint, focal cytoplasmic staining in less than } \\
50 \% \text { of tumor cells }\end{array}$ & Negative \\
\hline $2+$ & $\begin{array}{l}\text { Moderate, granular cytoplasmic staining } \\
\text { (can also partly present strong staining) in } \\
\text { more than 50\% of tumor cells }\end{array}$ & Positive \\
\hline $3+$ & $\begin{array}{l}\text { Strong, granular cytoplasmic staining in more } \\
\text { than 75\% of tumor cells, diffuse homogeneity } \\
\text { in distribution }\end{array}$ & Positive \\
\hline
\end{tabular}
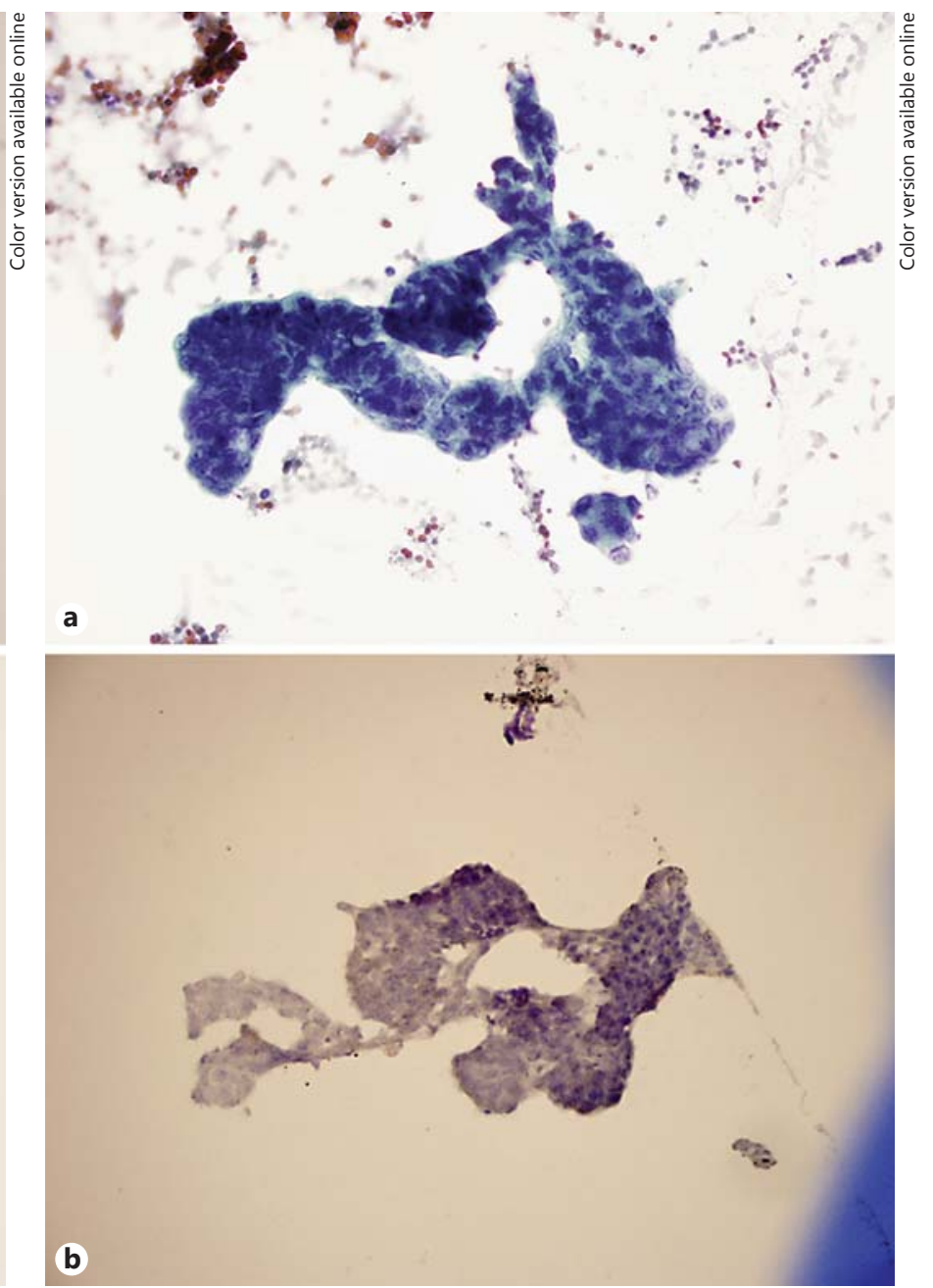

Fig. 2. Representative images of cases that were ALK ICC negative but FISH positive. a Papanicolaou-stained direct smears before CT. b ALK ICC staining using the D5F3 antibody. Original magnification: $\times 400$.

Table 2. Comparison between the results of ALK ICC and ALK FISH

\begin{tabular}{lllr}
\hline ALK ICC & $\begin{array}{l}\text { ALK FISH } \\
\text { positive }\end{array}$ & $\begin{array}{l}\text { ALK FISH } \\
\text { negative }\end{array}$ & Total \\
\hline Positive $(2+/ 3+)$ & $5(10.6)$ & $1(2.1)$ & $6(12.8)$ \\
$\begin{array}{l}\text { Negative }(0 / 1+) \\
\text { Total }\end{array}$ & $\begin{array}{l}2(4.3) \\
7(14.9)\end{array}$ & $\begin{array}{l}39(83.0) \\
40(85.1)\end{array}$ & $47(107.2)$ \\
\hline
\end{tabular}

Values are presented as numbers (\%). 


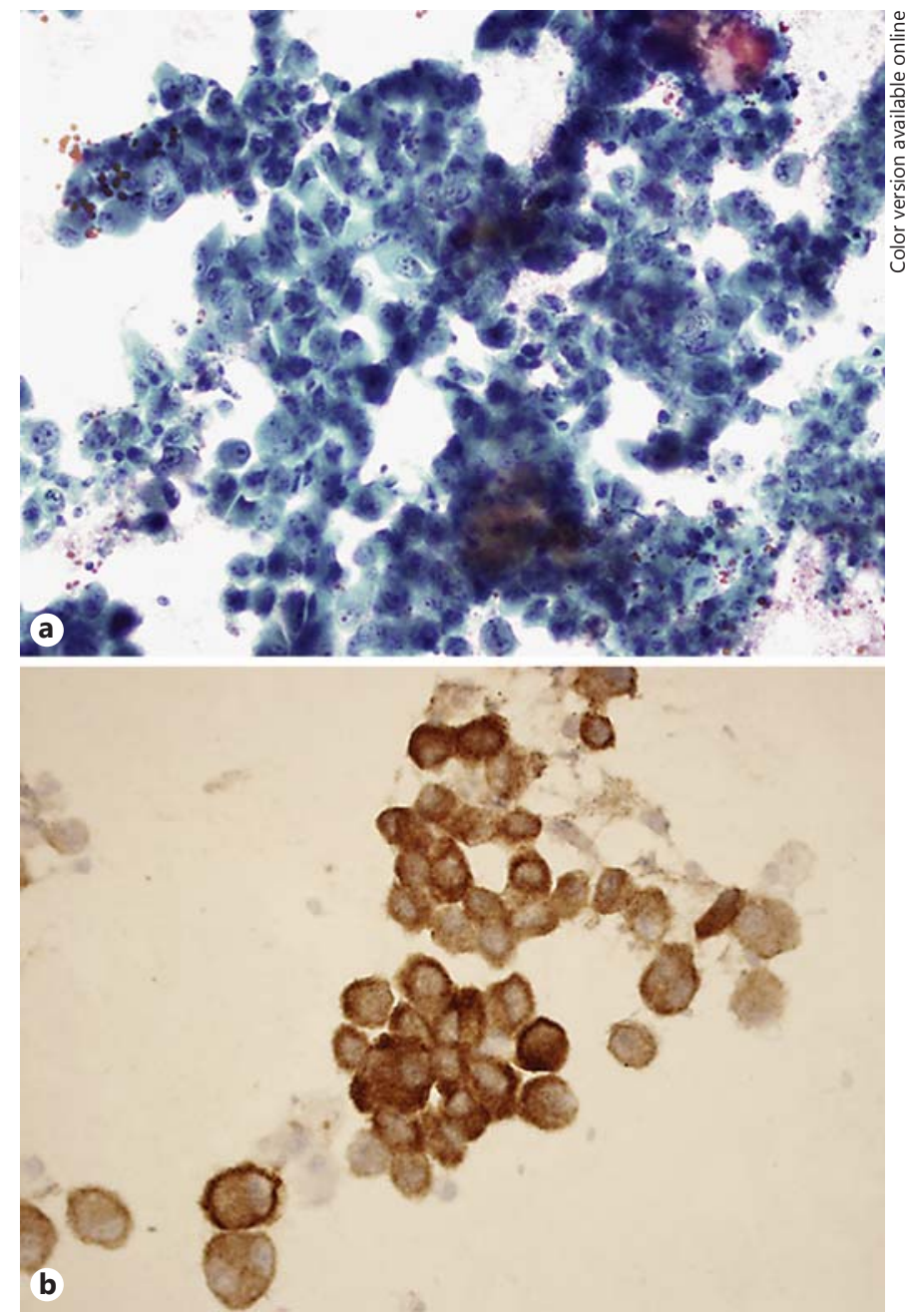

Fig. 3. Representative images of the case that was ALK ICC positive but FISH negative. a Papanicolaou-stained direct smears before CT. b ALK ICC staining using the D5F3 antibody. Original magnification: $\times 400$.

Thirty-nine of 40 FISH-negative cases (97.5\%) were negative on ALK ICC staining. All 39 ICC-negative cases showed no staining (score 0). One FISH-negative case showed strong ICC staining (score $3+$ ) on the CT smear (fig. 3 ) and the section cut from the CB also demonstrated 3+ expression for D5F3 immunostaining. The ALK FISH test for this case showed $0 \%$ tumor cells with ALK rearrangements and no sign of ALK gene copy number gain due to polysomy or amplification.

The negative predictive value of ALK ICC is 95\%. The overall correlation between ALK ICC and FISH was $93.6 \%$.

\section{Discussion}

Our study shows that the overall correlation between ALK FISH and ALK ICC using the CT technique is fairly strong (93.6\%) and the negative predictive value of ALK ICC is high (95\%). ALK rearrangement is a rare phenomenon in lung cancers, with a reported incidence rate of only about $3-5 \%$. As a result, the majority of samples received for ALK rearrangement testing will be negative. The high negative predictive value of ALK ICC makes it particularly useful for ALK rearrangement detection, since it may be used to screen out the majority of negative samples at a relatively high confidence level. The negative result by ALK ICC is even more reliable when other mutations such as EGFR mutations are identified in the same sample, since ALK rearrangements are almost mutually exclusive with other mutations.

Although ALK ICC has a fairly high negative predictive value, false negatives do exist. Two of 41 (5\%) ALK ICC-negative cases tested positive using FISH on concurrent biopsy tissue or $\mathrm{CB}$. Both of those cases had a low percentage (20 and $22 \%$ ) of tumor cells with ALK rearrangements on the FISH test, which may count as a possible cause of false negatives. According to the manufacturer's recommendation, $15 \%$ or more tumor cells with split signals are considered positive on ALK FISH assays. The actual percentage of tumor cells with ALK rearrangements is usually not reported. A recent study by Ilie et al. [14] compared FISH and IHC results on resected lung adenocarcinomas and found that all 5 discordant cases with an FISH+/IHC- profile had a low percentage of ALK+ tumor cells on FISH (15-20\%). It is conceivable that tumors with a low percentage of ALK+ tumor cells on FISH would express ALK protein at a lower level and/or in a more focal distribution compared to those with a high percentage of ALK+ tumor cells. Focal or weak expression of ALK protein may not be detected due to the limited cellularity of the transferred cytology smears. Both of our 2 false-negative cases had low to borderline cellularity on CT smears. Choosing more cellular smears would likely reduce or eliminate false-negative results in these cases; however, it is not always feasible. In our own practice, if the CB and the direct smears are both inadequate in cellularity, repeat sampling is recommended.

Another possible explanation for the false-negative result is rare ALK translocations that do not result in ALK protein overexpression or result in protein expression that is not recognized by this antibody clone. The study by Ilie et al. [14] showed that all $5 \mathrm{FISH}+/ \mathrm{IHC}$ - cases did 
not contain the most frequent EML4-ALK fusion transcripts as revealed by RT-PCR analysis.

The false-negative results were not likely due to a loss of antigenicity during the CT procedure, since the results of ICC performed on CT cytology smears showed high levels of agreement (97.5\%) with those of corresponding FFPE tissues in our previous study [12].

One case with strong staining on ICC was negative for ALK rearrangements by FISH on concurrent $\mathrm{CB}$, and IHC for ALK (D5F3) performed on the same CB also demonstrated 3+ staining. Previous studies have also reported rare cases in which ALK positivity by IHC is not reproduced by FISH. False-negative results of FISH may happen due to technical difficulties in the interpretation of results. For example, the splitting of the red and green signals can be extremely subtle, especially in interphase chromatin since the splitting of signals is a consequence of a paracentric inversion on the same chromosomal arm and the splitting signals are only about $12 \mathrm{Mb}$ apart. The percentage of ALK + tumor cells can be underestimated due to admixed normal cells. Another possible explanation is ALK protein overexpression caused by unknown types of ALK rearrangements or by events other than ALK rearrangements. An ALK gene copy number gain due to polysomy has been reported to cause ALK protein overexpression detected by IHC [14], although the false-positive case in our current study did not have evidence of an ALK gene copy number gain on the FISH test. Some authors have reported significant clinical improvement with crizotinib in patients with tumors that are ALK-negative by FISH but are found to be ALK-positive by IHC [15].

Our results are in agreement with those of previous studies using ICC to detect ALK on direct smears. Savic et al. [16] tested ALK ICC staining using the same clone (D5F3) on 41 Papanicolau-stained cytology slides after decolorization and found a high concordance rate compared to FISH results. Another smaller-scale study by Tanaka et al. [17] on 18 cases showed a high concordance rate between ALK ICC performed on destained direct smears and IHC performed on correlating FFPE tissue. Our study is different from these previous studies in that the CT technique is used prior to ICC. The CT technique has several advantages. The transferred smear can be divided into several parts so that multiple immunostains as well as multiple molecular tests can be performed from a single cellular smear. This is particularly important in the frequent situation in which only a few cellular smears are available, but there is a need to perform other diagnostic immunostains, such as TTF-1, to rule out metastatic adenocarcinoma from other primary sites. At our institu- tion, we routinely perform EGFR, KRAS molecular testing, and ALK ICC on the same cellular smear. For these patients, using the CT technique reduces the need for repeat FNA, thereby reducing potential patient morbidity and health care costs. Technically, CT is simple to perform and can be easily learned by a cytotechnologist or histotechnologist. There is no requirement for special equipment to perform this technique and the cost is relatively low. The only disadvantage of the CT technique is that at least $5 \mathrm{~h}$ of manual preparation are required to perform it, and this usually results in delay of the case for another day.

Although there is evidence that ALK IHC can be used as a screening test for the FISH test, we do not have enough evidence for using ALK ICC on CT smear as a screening test. In our current practice, the ALK FISH test performed on tissue or $\mathrm{CB}$ is still the first choice for detecting ALK rearrangements. ALK ICC is performed only when the CB lacks adequate cellularity. Clinicians are informed of the possibility of a false-negative ALK ICC test. Decisions of whether or not to repeat the biopsy are made considering the ALK ICC result together with other factors such as age, gender, smoking history, and EGFR/ KRAS mutation status.

The major limitation of the current study is its relatively small sample size, especially the small number of FISH-positive cases $(n=7)$. The common limitations of a retrospective study, such as selection bias and information errors, also exist. A prospective study with a large number of cases is needed for further characterization of the test.

In conclusion, our study showed that ICC performed on the FNA smears using the CT technique is an alternative method for the assessment of $A L K$ rearrangement, especially when the direct smears are highly cellular and the CB lacks adequate cellularity.

\section{Disclosure Statement}

There are no financial disclosures from any of the authors.
References
1 Kwak EL, Bang YJ, Camidge DR, et al: Ana- plastic lymphoma kinase inhibition in non- small-cell lung cancer. N Engl J Med 2010; 363:1693-1703. The biology and treatment of EML4-ALK non-small cell lung cancer. Eur J Cancer 2010; 46:1773-1780.
-2 Sasaki T, Rodig SJ, Chirieac LR, Janne PA: 
-3 Shaw AT, Kim DW, Nakagawa K, et al: Crizotinib versus chemotherapy in advanced ALKpositive lung cancer. N Engl J Med 2013;368: 2385-2394.

4 Camidge DR, Bang YJ, Kwak EL, et al: Activity and safety of crizotinib in patients with ALK-positive non-small-cell lung cancer: updated results from a phase 1 study. Lancet Oncol 2012;13:1011-1019.

5 Lindeman NI, Cagle PT, Beasley MB, et al: Molecular testing guideline for selection of lung cancer patients for EGFR and ALK tyrosine kinase inhibitors: guideline from the College of American Pathologists, International Association for the Study of Lung Cancer, and Association for Molecular Pathology. Arch Pathol Lab Med 2013;137:828-860.

6 Weickhardt AJ, Aisner DL, Franklin WA, Varella-Garcia M, Doebele RC, Camidge DR: Diagnostic assays for identification of anaplastic lymphoma kinase-positive non-small cell lung cancer. Cancer 2013;119:1467-1477.

7 Camidge DR, Kono SA, Flacco A, et al: Optimizing the detection of lung cancer patients harboring anaplastic lymphoma kinase (ALK) gene rearrangements potentially suitable for ALK inhibitor treatment. Clin Cancer Res 2010;16:5581-5590.
8 Zhou J, Zhao J, Sun K, et al: Accurate and economical detection of ALK positive lung adenocarcinoma with semiquantitative immunohistochemical screening. PLoS One 2014; 9:e92828.

9 Paik JH, Choe G, Kim H, et al: Screening of anaplastic lymphoma kinase rearrangement by immunohistochemistry in non-small cell lung cancer: correlation with fluorescence in situ hybridization. J Thorac Oncol 2011;6: 466-472.

10 Sherman ME, Jimenez-Joseph D, Gangi MD, Rojas-Corona RR: Immunostaining of small cytologic specimens: facilitation with cell transfer. Acta Cytol 1994;38:18-22.

$\checkmark 11$ Gong Y, Joseph T, Sneige N: Validation of commonly used immunostains on cell-transferred cytologic specimens. Cancer 2005;105: 158-164.

$12 \mathrm{Wu} \mathrm{HH}$, Jones KJ, Cramer HM: Immunocytochemistry performed on the cell-transferred direct smears of the fine-needle aspirates: a comparison study with the corresponding formalin-fixed paraffin-embedded tissue. Am J Clin Pathol 2013;139:754-758.
13 Ferguson J, Chamberlain P, Cramer HM, Wu HH: ER, PR, and Her2 immunocytochemistry on cell-transferred cytologic smears of primary and metastatic breast carcinomas: a comparison study with formalin-fixed cell blocks and surgical biopsies. Diagn Cytopathol 2013;41:575-581.

14 Ilie MI, Bence C, Hofman V, et al: Discrepancies between FISH and immunohistochemistry for assessment of the ALK status are associated with ALK 'borderline'-positive rearrangements or a high copy number: a potential major issue for anti-ALK therapeutic strategies. Ann Oncol 2015;26:238-244.

15 Sun JM, Choi YL, Won JK, et al: A dramatic response to crizotinib in a non-small-cell lung cancer patient with IHC-positive and FISHnegative ALK. J Thorac Oncol 2012;7:e36e38.

16 Savic S, Bode B, Diebold J, et al: Detection of ALK-positive non-small-cell lung cancers on cytological specimens: high accuracy of immunocytochemistry with the 5A4 clone. J Thorac Oncol 2013;8:1004-1011.

17 Tanaka H, Tone K, Hayashi A, et al: Clinical application of immunocytochemical detection of ALK rearrangement on cytology slides for detection or screening of lung adenocarcinoma. Lung Cancer 2013;80:289-292. 